\title{
1 A model of Zebrafish Avatar for co-clinical trials
}

2 Alice Usai ${ }^{1 \dagger}$, Gregorio Di Franco ${ }^{2, \dagger}$, Patrizia Colucci ${ }^{1}$, Luca Emanuele Pollina ${ }^{3}$,

3 Enrico Vasile ${ }^{4}$, Niccola Funel ${ }^{3}$, Matteo Palmeri², Luciana Dente ${ }^{1}$, Alfredo

4 Falcone $^{4}$, Luca Morelli2‡, Vittoria Raffa ${ }^{1 \ddagger^{*}}$

$5 \quad \dagger$ : Equally contributing

$6 \quad \ddagger$ : Equally contributing

$7 \quad$ *corresponding vittoria.raffa@unipi.it

8 1. Department of Biology, Università di Pisa, S.S. 12 Abetone e Brennero 4, 956127 Pisa, Italy.

102 . Department of Traslational Research and of New Surgical and Medical 11 Technologies, General Surgery Unit, University of Pisa, Via Paradisa 2, 56124 Pisa, 12 Italy.

13 3. Department of Surgical, Medical, Molecular Pathology and Critical Area,

14 Division of Surgical Pathology, University of Pisa,Via Paradisa 2, 56124 Pisa, 15 Italy.

164 4. Division of Medical Oncology, Pisa University Hospital, Via Roma 67, 56126 17 Pisa, Italy.

18 KEYWORDS patient-derived xenografts, zebrafish, chemosensitivity, 19 equivalent dose, translational research 
bioRxiv preprint doi: https://doi.org/10.1101/784041: this version posted September 26.2019 . The copyriaht holder for this preprint (which was not certified by peer review) is the author/funder, who has granted bioRxiv a license to display the preprint in perpetuity. It is made available under aCC-BY 4.0 International license.

21 The authors declare no conflicts of interest.

22 


\section{ABSTRACT}

Animal "Avatars" and co-clinical trials represent an emerging concept for implementing schemes of personalized medicine in oncology. In a co-clinical trial, the cancer cells of the patient tumor are xenotransplanted in the animal Avatar for drug efficacy studies and data collected in the animal trial are used to plan the best drug treatment in the patient trial. Recently, zebrafish has been proposed for implementing Avatar models but the lack of a general criterion for chemotherapy dose conversion from humans to fishes represents a limitation for conducting co-clinical trials.

Here, we validate a simple, reliant and cost-effective Avatar model based on the use of zebrafish larvae; by crossing data from safety and efficacy studies, we found a basic formula for the estimation of the dose to be used for running co-clinical trials and we validate it in a clinical study enrolling 24 adult patients with solid cancers (XenoZ, NCT03668418).

\section{ABBREVIATIONS}

5-FU, 5-Fluorouracil; dpf, days post fertilization; DMEM, Dulbecco's modified Eagle's medium; FBS, fetal bovine serum; ECF, 5-Fluorouracil + Cisplatin + Epirubicin; FLOT, 5-Fluorouracil + Lederfolin + Oxaliplatin + Docetaxel; FOLFIRI, 5-Fluorouracil + Lederfolin + Irinotecan; FOLFOX, 5-Fluorouracil + Lederfolin + Oxaliplatin; FOLFOXIRI, 5-Fluorouracil + Lederfolin + Oxaliplatin + Irinotecan; GEM, Gemcitabine; GEMCIS, Gemcitabine + Cisplatin; GEM/nab-P, Gemcitabine + nab-Paclitaxel; GEMOX, Gemcitabine + Oxaliplatin; hpf, hours post fertilization; hpi, hours post injection 


\section{INTRODUCTION}

Precision medicine refers to the approaches for tailoring a medical treatment to the individual characteristics of each patient (1). In particular, the "Mouse Avatar" is an emerging approach of precision medicine in oncology that has recently grown in importance (2); it implicates the xenotransplantation of cancer cells from patient tumor sample in mouse models to use them in drug efficacy studies. Mouse Avatars can be used to run "co-clinical trials" (3). In a co-clinical trial, the patient and murine trials are concurrently conducted and the drug efficacy response of the mouse study provides data to plan the best drug treatment of the patient tumor (4). The advantage of this approach is that each patient has his/her own tumor growing in an in vivo system, thereby allowing the identification of a personalized therapeutic approach. Nowadays, there are companies providing mouse Avatar generation and drug testing services to patients at a cost of tens thousands of dollars (5). The high cost is directly associated to the time-consuming process and the requirement of immunosuppressed strains (6). Unfortunately, this makes Avatars a cuttingedge technology available only for few people, posing a serious threat to the equal right to health for everyone. Recently, it has been proposed the use of zebrafish to make Avatars available for every patient and the approach sustainable for National Healthcare Systems. Zebrafish cancer models overcome the drawbacks of xenografts in mice (7). Zebrafish is highly fecund, develops rapidly and requires simple and inexpensive housing. Zebrafish embryos are transparent, allowing to image the engrafted cells in vivo, and they have high permeability to small molecules such as drugs used for chemotherapy. Last but not least they have low ethical impact when used in 
72 the larval stage from fecundation to 120 hours post fertilization (hpf) (8).

73 Zebrafish larvae as model for human cancer cell xenografts have been firstly

74 reported in 2005 (9). Since then, the use of zebrafish in vivo model of xenotransplantation has increased considerably (10). Several human cancer cells lines e.g. melanoma, glioma, adenocarcinoma, breast, pancreas and prostate cancer cell lines (11) as well as fragments of human cancer tissues (12) have been tested to date in zebrafish as engraftment host. Larvae provide a rejection-free permissive environment, where the xenotransplanted human cancer cells rapidly proliferate, migrate, form masses and induce neoangiogenesis, after injection (13). Most importantly, zebrafish larvae xenografts provide similar chemosensitive response of mouse xenografts (14).

However, in order to move forward in new paradigm of co-clinical trial using zebrafish Avatars, some critical aspects need to be solved. The biggest issue is related to the lack of the "equivalent dose" for translating the chemotherapy dosage used in humans to zebrafish larvae because one cannot apply the interspecies allometric approach for dose conversion from human to animal. The caveat is that chemotherapy drugs have to be administered in the fish water rather than injected as parenteral formulations. Therefore, drug safety and efficacy assessments are necessary to estimate the equivalent dose to administer (15). The present study aims to fill the gap regarding the dose conversion between zebrafish larvae and humans. A safety/efficacy study has been carried out in HCT 116 and MIA PaCa-2 cancer cell lines by testing 10 different chemotherapy regimens used in cancer treatment, i.e. FOLFOX (5Fluorouracil + Lederfolin + Oxaliplatin), FOLFIRI (5-Fluorouracil + Lederfolin + 
97 Irinotecan), FOLFOXIRI (5-Fluorouracil + Lederfolin + Oxaliplatin +

98 Irinotecan), ECF (5-Fluorouracil + Cisplatin + Epirubicin), FLOT (5-

99 Fluorouracil + Lederfolin + Oxaliplatin + Docetaxel), GEMCIS (Gemcitabine + 100 Cisplatin), Gem/nab-P (Gemcitabine + nab-Paclitaxel), GEMOX (Gemcitabine

$101+$ Oxaliplatin), Gemcitabine, 5-Fluorouracil. We found a general criterion for 102 dose equivalence that has been validated on zebrafish Avatar receiving fresh 103 tissue fragments taken from surgical specimens of patients underwent 104 surgical operation for hepato-biliary-pancreatic cancer and gastro-intestinal 105 cancer.

RESULTS

\section{Zebrafish safety study}

108 Dose-response analysis for the determination of the effects of chemotherapy 109 treatment on larvae was based on the evaluation of the phenotype resulting 110 from the exposure (i.e. normal, aberrant and dead). In particular, we exposed 111 larvae to 10 different chemotherapy treatments (GEM, GEMOX, GEM/nab-P, 112 GEMCIS, 5-FU, FOLFOX, FOLFIRI, FLOT, FOLFOXIRI, ECF, see 113 supplementary tables S1 and S2) for 72 hours, from 48 to 120 hpf (Figure 1).

114 Chemotherapy treatments induced death and a variety of malformations in 115 larvae, including yolk sac edema, pericardial edema and spine deformation. 116 For all regimens, deviation from phenotype without defect (normal phenotype) 117 increased with the increase of drug concentration. Linear regression analysis 118 showed an excellent relationship between the linear or logarithmic 119 concentration of the chemotherapy drug and the incidence of normal 120 phenotype $\left(R^{2}>0.95 ; p<0.05\right.$ for any protocol tested) or the incidence of 
121 mortality $\left(R^{2}>0.87 ; p<0.05\right.$ for any protocol tested), (Figure 1). For any

122 chemotherapy treatment, the dose that is lethal to $25 \%$ of the population

123 (LD25) and the concentration at which $50 \%$ of the normal phenotype is

124 inhibited (IC50) was determined (Figure 2A). Such data were also expressed 125 as Conversion Factor (CF):

126

$C F=\frac{E P C}{L C 25} \quad$ or $\quad C F=\frac{E P C}{I C 50}$

Eq.1

127 With EPC defined as human Equivalent Plasma Concentration, given by:

$128 \quad E P C=\frac{M}{V}$

$129 M$ being the total amount $(\mathrm{mg})$ of chemotherapy administered to humans by 130 the clinicians involved in the present study, $V(\mathrm{ml})$ being the mean volume of 131 human plasma (the EPC value for each regimen is given in table S2).

132 In the present study, we fixed the 75 percentiles of the box plots (Figure 2B) 133 as CF corresponding to the maximum tolerated doses (MTDs). Interestingly, 134 this value was similar for the 2 conditions, i.e. $C F=4.1$ and $C F=4.6$ for 135 EPC/LD25 and EPC/IC50, respectively. Consequently, the toxicity study 136 established a conversion factor $4.6 \leq \mathrm{CF}<\infty$ as the range for the 137 determination of the equivalent dose required for running co-clinical trials with 138 an acceptable safety level for the zebrafish trial.

139 Figure 1 Chemotherapy toxicity study. Zebrafish embryos 2 dpf were 140 incubated with media (E3 supplemented with $10000 \mathrm{U} / \mathrm{ml}$ penicillin and 100 $141 \mu \mathrm{g} / \mathrm{ml}$ streptomycin) modified with chemotherapy drugs or not modified at $14235^{\circ} \mathrm{C}$ for 3 days. At the end of the treatment the percentage of dead embryo 143 (red), aberrant (blue) and normal phenotype (green) was evaluated after 144 fixation and stereomicroscope observation. In GEM, GEMOX, GEM/nab-P, 
145 GEMCIS we reported the Gemcitabine concentration in the x-axis. In 5-FU, 146 FOLFOX, FOLFIRI, FLOT FOLFOXIRI, ECF we reported the 5-Fluorouracil 147 concentration in the $x$-axis. Control group showed an alteration from normal 148 phenotype $\leq 10 \%$. For each chemotherapy regimen a dose-response and the 149 relative linear regression analysis of the normal phenotype and the dead 150 embryos are shown. The resulting $R$ square is reported. The results 151 presented are a pool from three independent biological replicates $(n=90)$.

Figure 2 Estimation of the maximum tolerated dose. (A) Table and (B) box plot displaying EPC/IC50 EPC/LD25 ratios for all chemotherapy protocols.

\section{Zebrafish efficacy study}

For the estimation of the equivalent dose, we conducted an in vivo efficacy study based on human cancer cell lines, whose chemosensitivity has been already characterized in the literature. Specifically, 2 dpf larvae were xenotransplanted with Dil-stained human colorectal carcinoma cell line (HCT 116) or human pancreatic carcinoma cell line (MIA PaCa-2) into the yolk sac. To confirm the presence of the xenograft, injected larvae were screened by fluorescence microscopy 2 hours post injection (hpi). The screened larvae were randomly distributed in a multiwell plate (1 embryo/well) and equally divided among groups (control and chemotherapy regimens). In absence of chemotherapy, the Dil-stained area shows a statistically significant increase over the time (Figure 3, control group) and the block or the inversion of this tendency has been considered in the present study as a hallmark of chemotherapy effect. Indeed, we tested 4 chemotherapy regimens (5-FU, FOLFOX, FOLFOXIRI, FOLFIRI), which are the standard of care for the 
treatment of colorectal cancers, on HCT 116 cells xenotransplanted in $2 \mathrm{dpf}$ larvae. According to the toxicity study, we used conversion factors CF>4.6. First, a CF=8 was tested but data showed a statistically significant increase of the Dil-stained area at $1 \mathrm{dpi}$ and 2 dpi for all the regimens, suggesting the inefficacy of chemotherapy treatment at the CF used (Figure 3A). Therefore, we tested chemotherapy protocols at a higher concentration, corresponding to $\mathrm{CF}=5$. Interestingly, FOLFOXIRI were found to inhibit the increase of the stained area at $1 \mathrm{dpi}$ and $2 \mathrm{dpi}(\mathrm{p}>0.05)$, as opposite to the control, 5-FU, FOLFOX and FOLFIRI that showed a statistically significant progression (Figure 3B). The effect of FOLFOXIRI treatment was also confirmed by quantification of apoptosis in xenotransplanted (Dil-positive) cells revealing a significant increase of pyknotic nuclei with respect to the control group (no chemotherapy drugs) (Figure S1A).

The next step was to confirm the value of $\mathrm{CF}=5$ for dose equivalence by testing its efficacy on a different model, i.e. xenotransplanted larvae receiving MIA PaCa-2 cell line. Indeed, we tested 4 chemotherapy regimens (GEM, GEMOX, GEM-Nab, FOLFOXIRI), which are the standard of care for the treatment of pancreatic cancers, on MIA PaCa-2 cells xenotransplanted in 2 dpf larvae. GEM and GEM-Nab-P proved to be the most efficient regimens, with no statistically significant increase of the Dil-stained area at $1 \mathrm{dpi}$ and 2 dpi, as opposite to the control, GEMOX and FOLFOXIRI (Figure 3C).

Figure 3 Efficacy analysis. Evaluation of the effects of chemotherapy on cancer cell lines (HCT 116, MIA PaCa-2) xenotransplanted in 2dpf zebrafish embryos. Each embryo was imaged at 2 hpi, 1 dpi, 2 dpi and the relative area is the Dil-stained area normalized with respect to the 2 hpi time point. (A) 
194 Chemosensitivity of HCT 116 xenografts, CF=8. A statistically significant 195 increase of relative area was observed in all groups. (B) Chemosensitivity of 196 HCT 116 xenografts, $C F=5$. A statistically significant increase of relative area 197 was observed in control, 5-FU, FOLFOX and FOLFIRI but not in FOLFOXIRI.

198 (C) Chemosensitivity of MIA PaCa-2 xenografts, CF=5. A statistically 199 significant increase of relative area was observed in control, GEMOX and FOLFOXIRI treatments but not in GEM and GEM/nab-P. Data are mean \pm

201 SEM and representative of three independent assays. $n \geq 15$ (embryos), 2-way 202 ANOVA followed by Bonferroni correction (all groups compared against $k$ 203 group). ${ }^{*} p<0.05 ;{ }^{* *} p<0.01 ;{ }^{* * *} p<0.001 ;{ }^{* * * *} p<0.0001$.

\section{Zebrafish Avatar}

A total of 6 patients operated for adenocarcinoma of the colon $(n=3)$, pancreatic ductal adenocarcinoma $(n=1)$, and gastric adenocarcinoma $(n=2)$ have been enrolled in the study (NCT03668418) to establish the zebrafish Avatar model. In order to preserve the tumor micro-environment, we decided to xenotransplant fresh tissue fragments screened by the histopathology unity of the Azienda Ospedaliera Universitaria Pisana, by modifying the protocol

211 published by Marques et al. (12). Briefly, the tissue was Dil/DiO-stained, 212 disaggregated using Dumont forceps (No.5) into a relative size of $1 / 2-1 / 4$ the 213 size of the yolk and xenotransplanted in the yolk of $2 \mathrm{dpf}$ larvae. After 214 transplantation, larvae were incubated for $2 \mathrm{~h}$ at $35^{\circ} \mathrm{C}$, then screened to check 215 for presence of the stained tissue and imaged at $2 \mathrm{hpi}, 1 \mathrm{dpi}$ and $2 \mathrm{dpi}$. Cell 216 engraftment was also confirmed by histological analysis performed at $2 \mathrm{dpi}$. 217 Hoechst staining documents healthy cell nuclei (Figure S1B) and H\&E 218 staining shows the presence of cancer cells that have the typical round-shape 
219 morphology with large nuclei (Figure S2A). Interestingly, H\&E staining performed on zebrafish embryos xenotransplanted with fragments of normal tissue taken from normal mucosa or pancreatic parenchyma of the surgical specimen do not show any cell with typical cancer morphology but exclusively cells with a typical fibroblast-like shape (Figure S2B). In order to perform the analysis, we measured the size of the region of interest (ROI) corresponding to the stained area at $2 \mathrm{hpi}, 1 \mathrm{dpi}$ and $2 \mathrm{dpi}$ (Figure 4A3-C3). The mean size of the tumor mass area measured in each time point was normalized with respect to the 2 hpi time point. We found an increase of the stained area versus time in all cases, which was statistically significant at 2 dpi with respect 229 to the time point 2 hpi for 5 patients of 6 (83\%, Figure 5). According to this 230 finding, the measure of the size of the relative stained area at $2 \mathrm{dpi}$ has been 231 fixed as primary measure of the study. Sporadically we also detected cancer 232 cell migration (Figure S3).

Figure $4 \mathrm{~A}$ representative larva xenotransplanted with a fresh tumor specimen of gastric cancer (patient S013). Bright-field images of the grafted larvae (A1-C1), epi-fluorescence images (A2-C2) and overlay (A3-C3), showing the region of interest (ROI; yellow line). All images are oriented so that rostral end is on the left and dorsal end is on the top.

Figure 5 Quantitative analysis of six cases of patient-derived tumor xenografts. Dil-stained area at time point 2 hpi, 1 dpi and 2 dpi was 240 normalized with respect to the time point 2 hpi. Patient enrollment code is 241 reported $(\mathrm{C}=$ Colon, $P=$ Pancreas, $S=$ Stomach) and the number of embryos 
as mean \pm SEM. ${ }^{*} p<0.05 ;{ }^{* *} p<0.01$ by 1 - way ANOVA followed by

\section{Zebrafish trial}

24 adult patients with pancreatic cancers $(n=12)$, colon cancer $(n=8)$ and

247 gastric cancers $(n=4)$ undergoing a chemotherapy treatment have been

248 recruited for this part of the study. After surgery and histopathology screening, 249 patient biopsies have been xenotransplanted in 100 zebrafish embryos and 250 injected embryos were randomly allocated among 5 groups (4 therapeutic 251 options and one control group). Groups were exposed to all chemotherapy 252 options, according to the cancer type, by dissolving the chemotherapy in fish 253 water, according to the equivalent dose corresponding to $C F=5$. Two days 254 post treatment the response of zebrafish xenografts to the chemotherapy 255 options was analyzed by monitoring the ROI size at $2 \mathrm{hpi}, 1 \mathrm{dpi}$ and $2 \mathrm{dpi}$ 256 (Figure S4). The chemotherapy protocols tested were 5-FU, FOLFOX, 257 FOLFIRI and FOLFOXIRI for colon cancer; GEM, GEMOX, GEM/nab-P and 258 FOLFOXIRI for pancreatic case and FOLFOX, FOLFORI, FLOT and ECF for gastric cancer. We adapted the "Response evaluation criteria in solid tumors 260 (RECIST)" to the fish trial by defining the partial response (PR, at least a 30\% 261 decrease in the relative stained area at $2 \mathrm{dpi} / 2 \mathrm{hpi}$, taking as reference the 262 relative stained area at $2 \mathrm{dpi} / 2 \mathrm{hpi}$ of the control group) and complete 263 response (CR, at least a 90\% decrease in the relative stained area at $2 \mathrm{dpi} / 2$ $264 \mathrm{hpi}$, taking as reference the relative stained area at $2 \mathrm{dpi} / 2 \mathrm{hpi}$ of the control group) (Figure 6). For patients affected by colon cancer, we observed a PR in 
number of patients (12.5\%) and only to FOLFIRI chemotherapy. For patients

269 affected by pancreatic cancer, we observed a PR to GEM/nab-P $(58.33 \%$ of

270 patients), GEM (50\%), GEMOX (50\%), a limited PR to FOLFOXIRI (33.33 \%)

271 but we never observed CR for any chemotherapy treatment. For patients

272 operated for gastric cancer, we observed high incidence of PR to FOLFIRI 273 (100\% of patients) but low incidence of PR to FOLFOX, FLOT and ECF (25\%

274 of patients); we also observed CR to FOLFIRI in one patient of four.

Figure 6 Percentage of Partial Response (PR) and Complete Response

(CR). FOLFOXIRI, FOLFIRI, FOLFOX and 5-FU treatments in Zebrafish

Avatar xenotransplanted with colon tumor ( $n=8$ patient case analyzed) (A);

278 GEMOX, GEM/nab-P, GEM, FOLFOXIRI treatments in Zebrafish Avatar

xenotransplanted with pancreas tumor ( $n=12$ patient case analyzed) (B); ECF,

FLOT, FOLFIRI and FOLFOX treatments in Zebrafish Avatar

xenotransplanted with gastric tumor ( $n=4$ patient case analyzed) (C).

282

283

284

285

286 $289 \quad(p=0.04)$.

Interestingly, the zebrafish Avatar can be used to perform chemosensitivity assessment on a single patient basis. Four representative case of patient enrolled in the study are given in Figure 7. As for the two cases of colon cancer, we could observe significant response $(p=0.03)$ to FOLFOXIRI treatment in patient C024, and to 5-FU $(p=0.05)$ and FOLFIRI $(p=0.02)$ in patient C031. As for the two cases of pancreatic cancer, FOLFOXIRI proved to be the efficient regimen in patient P025 $(p=0.02)$ and in patient P030

290 Figure 7 Chemosensitivity assay. 48 hpf embryos were injected with 291 fragments of patient's tumor tissue and treated for 48 hours with different 
chemotherapy compounds at the $C F=5$. Representative cases of colon cancer

293 (patient enrolled code: C024, C031) and pancreatic cancer (patient enrolled

294 code: P025, P030) with quantitative analysis of relative tumor area (2 dpi/2 hpi

295 for colon and 2 dpi/24 hpi for pancreas). All graphs show an increase of the

296 stained area over the time in control group. The cases C024, P025, P030

297 show a statistically significant regression of the stained area size in

298 FOLFOXIRI treated group. The case C031 shows significant stained area

299 reduction in 5-FU and FOLFIRI treated groups. Results are expressed as

300 mean \pm SEM and analyzed by 1-way ANOVA followed by Dunnett's multiple

301 comparisons test. * $p<0.05, n \geq 3$.

314 from human to fish need to be identified.

315 In this work, we found a general dose conversion criterion based on the 316 following formula: 
$317 \quad$ Cfish $=\frac{M}{V} / C F$

Eq.3

318 Where $c_{\text {fish }}(\mathrm{mg} / \mathrm{ml})$ is the chemotherapy concentration in fish water, $M$ is the 319 total amount $(\mathrm{mg})$ of chemotherapy administered to humans, $V(\mathrm{ml})$ is the 320 volume of human plasma and $C F$ is the conversion factor that we estimated to 321 be $C F=5$. We estimated this value by matching data collected from the safety and efficacy studies performed in zebrafish. The safety study was performed on WT larvae. The efficacy study was performed on larvae xenotransplanted with human cancer cell line whose response to chemotherapy has been already characterized. Specifically, we found that HCT 116 responded to 326 FOLFOXIRI treatment with higher sensitivity, but not to 5-FU, FOLFOX and 327 FOLFIRI at the CF proposed. These results are concordant with the literature suggesting that first-line FOLFOXIRI chemotherapy leads to improved survival and efficacy of metastatic colorectal cancer patient outcomes in comparison 330 to FOLFIRI or FOLFOX chemotherapy (22). We also tested the response of 331 MIA PaCa-2 cells by observing high sensitivity to GEM and GEM/nab-P treatments. This analysis is confirmed by the efficacy data from metastatic pancreatic cancer patients treated with GEM/nab-P (23). Such experimental evidences, obtained by testing the efficacy of chemotherapy on two cancer cell lines from different types of human tumor (colorectal and pancreatic), 336 suggest that the selected therapeutic dose (corresponding to $C F=5$ ) is 337 effective in killing tumor cells and, in principle, predictive of the best 338 pharmacological treatment. Indeed, we suggest the use of the conversion 339 factor $C F=5$ in any co-clinical trial using zebrafish Avatars. This represents a 340 starting point for any further research step that aims to validate the zebrafish 341 Avatars as valuable tools to support the oncologists in the clinical routine. 
342 Potential applications are the evaluation of the disease prognosis and

343 chemosensitivity assays for the prediction of the most effective chemotherapy

344 scheme. Indeed, we validated an approach consisting in the

345 xenotransplantation of pieces of the patient tumor tissue, after surgery and

346 histopathology screening, in order to obtain a model for testing the response

347 of the patient tumor to the different chemotherapy regimens, with an

348 assessment in less than one week (Figure S4). The xenotransplantation of

349 cancer cells isolated and propagated from patient tumors in zebrafish is an

350 approach more popular than the xenotransplantation of tissue fragments.

351 Unfortunately, isolated cancer cells tend to lose cell heterogeneity and the

352 stromal contribution. Moreover, during the process of isolation and adaptation,

353 clones with a higher proliferative rate than that of the primary tumor are

354 selected and thus they could not be representative of the cancer cell

355 population (24). Indeed, for precision medicine and personalized medicine,

356 the xenotransplantation of biopsy or surgical specimen fragments screened by

357 the pathologist would be recommended to develop patient-derived xenografts

358 in which the stromal counterpart and cancer cell heterogeneity are both

359 preserved (25). Our data suggest that fresh tumor tissue transplanted in $2 \mathrm{dpf}$

360 larvae can engraft and survive in the host, as documented by histological

361 analysis showing typical cancer cell morphology (H\&E staining, Figure S2A)

362 and absence of pyknotic nuclei (Hoechst staining, Figure S1B). The survival

363 rate of the xenografted host was acceptable, at both $1 \mathrm{dpi}(81 \%, \mathrm{n}=101)$ and

364 at 2 dpi $(68 \%, n=101)$. We also detected the capacity of cancer cell

365 extravasion and dissemination in distal tissues (Figure S3). As the relative

366 area at $2 \mathrm{dpi} / 2 \mathrm{hpi}$ has been fixed as primary measure of the study, we 
performed the efficacy tests under the assumption that a statistically significant decrease of this measure with respect to control group (no chemotherapy) is a hallmark of chemotherapy response. Specifically, we tested chemosensitivity in 24 human tumor fragments taken from surgical specimen. To this purpose, pieces of tumor tissue were microinjected in zebrafish embryos to create Zebrafish Avatar and treated with chemotherapy drugs at a concentration corresponding to $C F=5$. Interestingly, our experimental data have shown good agreement with observations registered in the common clinical practice. In fact, for patients affected by colon cancer (Figure 6A), we found a superiority of the chemotherapy treatment when a combination of drugs are used (FOLFOX, FOLFIRI and FOLFOXIRI) respect to the use of only 5-FU (26). Additionally, we found a similar response to FOLFOX and FOLFIRI (27). The higher aggressiveness of pancreatic cancers associated with a lower response to chemotherapy compared to colon and gastric cancers may be the reason why a complete response was never observed in our experiments for this group of patients (Figure 6B). For the group of patients affected by gastric cancer (Figure 6C), we found an excellent response to FOLFIRI that can be considered an acceptable first-line treatment for advanced gastric cancers (28).

Interestingly, the use of zebrafish Avatars allows to appreciate a different response to different chemotherapy regimens on a single-patient basis (Figure 7). Further tests will be necessary to fully validate the zebrafish Avatar here proposed as a clinical tool predictive of the most effective treatment for each patient. Future experiments will be devoted to enroll in the study a higher number of cases in order to correlate the chemosensitivity results 
392

393

394

obtained in the animal trial with the response to the chemotherapy treatment observed in the human trial.

\section{MATERIALS AND METHODS}

\section{Zebrafish husbandry}

Zebrafish (Danio rerio) were handled in compliance with local animal welfare regulations (authorization n. 99/2012-A, 19.04.2012; authorization for zebrafish breeding for scientific purposes released by the "Comune di Pisa" DN-16/43, 19/01/2015) and standard protocols approved by Italian Ministry of Public Health, in conformity with the Directive 2010/63/EU. Zebrafish fertilized eggs were obtained by natural mating of wild-type fishes at our facilities and the developing embryos were staged in incubator at $28^{\circ} \mathrm{C}$ according to Kimmel et al. (29). Before any procedure, embryos were anesthetized in $0.02 \%$ tricaine.

\section{Cell culture, staining and microinjections}

The HCT 116 human colorectal carcinoma cells were cultured in McCoy's 5A Modified Medium supplemented with 10\% fetal bovine serum (FBS), 10000 $\mathrm{U} / \mathrm{ml}$ penicillin and $100 \mu \mathrm{g} / \mathrm{ml}$ streptomycin. The Mia Paca-2 human pancreatic carcinoma cells were cultured in DMEM supplemented with 10\% FBS, 10000 $\mathrm{U} / \mathrm{ml}$ penicillin and $100 \mu \mathrm{g} / \mathrm{ml}$ streptomycin. Cells were incubated at $37^{\circ} \mathrm{C}$ with $5 \%$ of $\mathrm{CO}_{2}$ in humidified atmosphere. Cells were detached at $80 \%$ confluence with $0.25 \%(w / v)$ trypsin- $0.53 \mathrm{mM}$ EDTA solution and stained with $10 \mu \mathrm{g} / \mathrm{ml}$ CM-Dil for $15 \mathrm{~min}$ at $37^{\circ} \mathrm{C}$ followed by $15 \mathrm{~min}$ on ice in darkness. Cells were washed and centrifuged three times by D-PBS and resuspended in D-PBS supplemented with $10 \%$ FBS to a final concentration of 100 cells/nl. All the 
reagents were supplemented by Thermo Fisher Scientific, Waltham, MA.

Dechorionated embryos at 2 days post fertilization (dpf) were anesthetized and injected with four nanoliters of cells suspension in the left side of the perivitelline space using a heat-pulled needle and the PV830 Pneumatic

420 PicoPump microinjector. The embryos were incubated at $35^{\circ} \mathrm{C}$, and one hours 421 after injection were screened with fluorescence microscope.

The clinical study was approved by the "Comitato Etico Regionale per la

OVEST" (09/11/2017, prot n 70213). Human material from surgical resected specimens was obtained from the Azienda Ospedaliera Pisana (Pisa, Italy) after written informed consent of the patients and approval of local Ethical Committee. Tumor tissue screened by the histopathologist (from supplemented with $10000 \mathrm{U} / \mathrm{ml}$ penicillin, $100 \mu \mathrm{g} / \mathrm{ml}$ streptomycin and 100 $431 \mu \mathrm{g} / \mathrm{ml}$ Amphotericin and cut into small pieces (1-3 $\mathrm{mm})$ using a scalp blade. 432 The pieces were then transferred to a $5 \mathrm{ml}$ tube, and stained with either 40 $433 \mu \mathrm{g} / \mathrm{ml}$ DiO in D-PBS (in case of esophageal and gastric cancers) or $40 \mu \mathrm{g} / \mathrm{ml}$ 434 CM-Dil in D-PBS (in case of hepato-biliary-pancreatic cancers and intestinal 435 cancers). The tissue pieces were incubated for $15 \mathrm{~min}$ at $37^{\circ} \mathrm{C}$ and $15 \mathrm{~min}$ in 436 ice cube. Tissue pieces were then washed and centrifuged three times by D437 PBS and resuspended in D-PBS supplemented with $10 \%$ FBS. For tissue 438 transplantation we used the manual method proposed by Marques et al. 2009 439 (12). In particular before transplantation, small pieces of stained tissue were 440 further disaggregated using Dumont forceps (No.5) into a relative size of $1 / 4$ 
441 to $1 / 2$ the size of the yolk. Tissue pieces with the correct size were transferred

442 to $1 \%$ agarose disks in multiwell plates in which the 2 dpf embryos were 443 laying, ready for transplantation. A glass transplantation needle was used to 444 transfer the tissue into the yolk. The tissue was picked up, put on top of the 445 yolk and then pushed inside. The yolk usually sealed itself and in the majority 446 of embryos, the tumor remained in the yolk. After transplantation, embryos 447 were incubated for $2 \mathrm{~h}$ at $35^{\circ} \mathrm{C}$, then embryos were checked for presence of 448 tissue and incubated at $35^{\circ} \mathrm{C}$ in $\mathrm{E} 3$ supplemented with $10000 \mathrm{U} / \mathrm{ml}$ penicillin 449 and $100 \mu \mathrm{g} / \mathrm{ml}$ streptomycin with the presence or absence of drugs for the 450 following days in the respect of the treatment plan.

\section{Anticancer drugs toxicity and treatment plan}

452 Groups of 30 embryos (2 dpf) arrayed in multiwell plates were exposed to E3 453 supplemented with $10000 \mathrm{U} / \mathrm{ml}$ penicillin and $100 \mu \mathrm{g} / \mathrm{ml}$ streptomycin 454 unmodified (control) and modified with the chemotherapy drug at $35^{\circ} \mathrm{C}$ for $24 \mathrm{~h}$ 455 added with increasing concentrations (Tables S1, S2).

456 The drugs were refreshed each day for the three days of treatment plan. 3 457 days after treatment (3 dpt) zebrafish larvae were fixed in $4 \%$ 458 paraformaldehyde in PBS at $4^{\circ} \mathrm{C}$ over night. After that, they were dehydrated 459 with increasing concentration of ethanol, and analyzed by stereo microscope 460 to evaluate the phenotype (normal, death, aberrant).

462 Two hours post injection (2 hpi) zebrafish embryos xenotransplanted with 463 cancer cell lines were anesthetized with $0.02 \%$ tricaine and positioned 464 laterally, with the site of the implantation to the top. The embryos were imaged 
by fluorescence microscope and transferred to a 24-well plate (one embryo/well) containing chemotherapy compounds in E3 supplemented with $10000 \mathrm{U} / \mathrm{ml}$ penicillin and $100 \mu \mathrm{g} / \mathrm{ml}$ streptomycin or E3 supplemented with $10000 \mathrm{U} / \mathrm{ml}$ penicillin and $100 \mu \mathrm{g} / \mathrm{ml}$ streptomycin unmodified (control). All

469 embryos were imaged everyday during the time course of the treatment. The size of the tumor area was measured by using ImageJ.

At 2 dpi (4 dpf) the xenografted larvae were fixed in 4\% paraformaldehyde for eosin staining or OCT embedding for Hoechst staining. Larvae were

475 respectively sectioned with microtome or cryostat, along the sagittal plane at a 476 thickness of $8 \mu \mathrm{m}$.

477 Histopathological analysis was performed on paraffin sections stained by 478 hematoxylin \& eosin (Merck KGaA, Germany) and digitally imaged using 479 Nikon Eclipse E600 microscope.

480 Cryostat sections were Hoechst 33342 counterstained. Digital images of the 481 stained sections were generated using a Nikon Eclipse Ti. Pyknotic cells was 482 counted at $40 \mathrm{X}$ magnification within the epifluorescence DAPI image.

\section{Statistical analysis}

484 We used GraphPad Prism 7 as statistical analysis software. Data analysis was performed by ANOVA, followed by Bonferroni correction or Dunnett's post-hoc test or t-test. Statistical significance was set to $5 \%$.

488 The authors thank Ida Montesanti and Noemi Nardillo for supporting zebrafish 
imaging and Dr Martina Giannaccini for the experimental advices. This work

was supported by Fondazione Pisa (project 114/16).

\section{REFERENCES}

1. Millner LM, Strotman LN. The Future of Precision Medicine in Oncology. Clinics in laboratory medicine. 2016 Sep;36(3):557-73. PubMed PMID: 27514468.

2. Hidalgo M, Amant F, Biankin AV, Budinska E, Byrne AT, Caldas C, et al. Patient-derived xenograft models: an emerging platform for translational cancer research. Cancer discovery. 2014 Sep;4(9):998-1013. PubMed PMID: 25185190. Pubmed Central PMCID: 4167608.

3. Clohessy JG, Pandolfi PP. Mouse hospital and co-clinical trial project--from bench to bedside. Nature reviews Clinical oncology. 2015 Aug;12(8):491-8. PubMed PMID: 25895610.

4. Malaney P, Nicosia SV, Dave V. One mouse, one patient paradigm: New avatars of personalized cancer therapy. Cancer letters. 2014 Mar 1;344(1):1-12. PubMed PMID: 24157811. Pubmed Central PMCID: 4092874.

5. Schmidt C. New mouse models mimic biology of human cancer. J Natl Cancer Inst. 2015;107(2). PubMed PMID: Medline:25677039. English.

6. Tentler JJ, Tan AC, Weekes CD, Jimeno A, Leong S, Pitts TM, et al. Patientderived tumour xenografts as models for oncology drug development. Nature reviews Clinical oncology. 2012 Apr 17;9(6):338-50. PubMed PMID: 22508028. Pubmed Central PMCID: 3928688.

7. $\quad$ Teng Y, Xie X, Walker S, White DT, Mumm JS, Cowell JK. Evaluating human cancer cell metastasis in zebrafish. BMC cancer. 2013 Oct 4;13:453. PubMed PMID: 24089705. Pubmed Central PMCID: 3852235.

8. Giannaccini M, Cuschieri A, Dente L, Raffa V. Non-mammalian vertebrate embryos as models in nanomedicine. Nanomedicine : nanotechnology, biology, and medicine. 2014 May;10(4):703-19. PubMed PMID: 24103306.

9. Lee LM, Seftor EA, Bonde G, Cornell RA, Hendrix MJ. The fate of human malignant melanoma cells transplanted into zebrafish embryos: assessment of migration and cell division in the absence of tumor formation. Developmental dynamics : an official publication of the American Association of Anatomists. 2005 Aug;233(4):1560-70. PubMed PMID: 15968639.

10. Jackson SJ, Thomas GJ. Human tissue models in cancer research: looking beyond the mouse. Disease models \& mechanisms. 2017 Aug 1;10(8):939-42. PubMed PMID: 28768734. Pubmed Central PMCID: 5560067.

11. Barriuso J, Nagaraju R, Hurlstone A. Zebrafish: a new companion for translational research in oncology. Clinical cancer research : an official journal of the American Association for Cancer Research. 2015 Mar 1;21(5):969-75. PubMed PMID: 25573382. Pubmed Central PMCID: 5034890. 
12. Marques IJ, Weiss FU, Vlecken DH, Nitsche C, Bakkers J, Lagendijk AK, et al. Metastatic behaviour of primary human tumours in a zebrafish xenotransplantation model. BMC cancer. 2009 Apr 28;9:128. PubMed PMID: 19400945. Pubmed Central PMCID: 2697170.

13. Haldi M, Ton C, Seng WL, McGrath P. Human melanoma cells transplanted into zebrafish proliferate, migrate, produce melanin, form masses and stimulate angiogenesis in zebrafish. Angiogenesis. 2006;9(3):139-51. PubMed PMID: 17051341.

14. Fior R, Povoa V, Mendes RV, Carvalho T, Gomes A, Figueiredo N, et al. Single-cell functional and chemosensitive profiling of combinatorial colorectal therapy in zebrafish xenografts. Proceedings of the National Academy of Sciences of the United States of America. 2017 Sep 26;114(39):E8234-E43. PubMed PMID: 28835536. Pubmed Central PMCID: 5625889.

15. Nair $A B$, Jacob $S$. A simple practice guide for dose conversion between animals and human. Journal of basic and clinical pharmacy. 2016 Mar;7(2):27-31. PubMed PMID: 27057123. Pubmed Central PMCID: 4804402.

16. Braekeveldt N, Bexell D. Patient-derived xenografts as preclinical neuroblastoma models. Cell and tissue research. 2018 May;372(2):233-43. PubMed PMID: 28924803. Pubmed Central PMCID: 5915499.

17. Mery B, Rancoule C, Guy JB, Espenel S, Wozny AS, Battiston-Montagne P, et al. Preclinical models in HNSCC: A comprehensive review. Oral oncology. 2017 Feb;65:51-6. PubMed PMID: 28109468.

18. Krempley $\mathrm{BD}, \mathrm{Yu} \mathrm{KH}$. Preclinical models of pancreatic ductal adenocarcinoma. Chinese clinical oncology. 2017 Jun;6(3):25. PubMed PMID: 28705002.

19. Gengenbacher N, Singhal M, Augustin HG. Preclinical mouse solid tumour models: status quo, challenges and perspectives. Nature reviews Cancer. 2017 Dec;17(12):751-65. PubMed PMID: 29077691.

20. Day CP, Merlino G, Van Dyke T. Preclinical mouse cancer models: a maze of opportunities and challenges. Cell. 2015 Sep 24;163(1):39-53. PubMed PMID: 26406370. Pubmed Central PMCID: 4583714.

21. Taylor AM, Zon LI. Zebrafish tumor assays: the state of transplantation. Zebrafish. 2009 Dec;6(4):339-46. PubMed PMID: 20047467. Pubmed Central PMCID: 2809423.

22. Marques RP, Duarte GS, Sterrantino C, Pais HL, Quintela A, Martins AP, et al. Triplet (FOLFOXIRI) versus doublet (FOLFOX or FOLFIRI) backbone chemotherapy as first-line treatment of metastatic colorectal cancer: A systematic review and meta-analysis. Critical reviews in oncology/hematology. 2017 Oct;118:5462. PubMed PMID: 28917269.

23. Tehfe M, Dowden S, Kennecke H, El-Maraghi R, Lesperance B, Couture F, et al. nab-Paclitaxel Plus Gemcitabine Versus Gemcitabine in Patients with Metastatic Pancreatic Adenocarcinoma: Canadian Subgroup Analysis of the Phase 3 MPACT Trial. Advances in therapy. 2016 May;33(5):747-59. PubMed PMID: 27085323. Pubmed Central PMCID: 4882352. 
24. Barriuso J, Nagaraju R, Hurlstone A. Zebrafish: a new companion for translational research in oncology. Clinical cancer research : an official journal of the American Association for Cancer Research. 2015;21(5):969-75. PubMed PMID: Medline:25573382. English.

25. Mercatali L, La Manna F, Groenewoud A, Casadei R, Recine F, Miserocchi G, et al. Development of a Patient-Derived Xenograft (PDX) of Breast Cancer Bone Metastasis in a Zebrafish Model. Int J Mol Sci. 2016;17(8). PubMed PMID: Medline:27556456. English.

26. Giacchetti S, Perpoint B, Zidani R, Le Bail N, Faggiuolo R, Focan C, et al. Phase III multicenter randomized trial of oxaliplatin added to chronomodulated fluorouracil-leucovorin as first-line treatment of metastatic colorectal cancer. Journal of clinical oncology : official journal of the American Society of Clinical Oncology. 2000 Jan;18(1):136-47. PubMed PMID: 10623704.

27. Tournigand C, Andre T, Achille E, Lledo G, Flesh M, Mery-Mignard D, et al. FOLFIRI followed by FOLFOX6 or the reverse sequence in advanced colorectal cancer: a randomized GERCOR study. Journal of clinical oncology : official journal of the American Society of Clinical Oncology. 2004;22(2):229-37. PubMed PMID: Medline:14657227. English.

28. Guimbaud R, Louvet C, Ries P, Ychou M, Maillard E, Andre T, et al. Prospective, randomized, multicenter, phase III study of fluorouracil, leucovorin, and irinotecan versus epirubicin, cisplatin, and capecitabine in advanced gastric adenocarcinoma: a French intergroup (Federation Francophone de Cancerologie Digestive, Federation Nationale des Centres de Lutte Contre le Cancer, and Groupe Cooperateur Multidisciplinaire en Oncologie) study. Journal of clinical oncology : official journal of the American Society of Clinical Oncology. 2014 Nov 1;32(31):35206. PubMed PMID: 25287828.

29. Kimmel CB, Ballard WW, Kimmel SR, Ullmann B, Schilling TF. Stages of embryonic development of the zebrafish. Developmental dynamics : an official publication of the American Association of Anatomists. 1995 Jul;203(3):253-310. PubMed PMID: 8589427. 

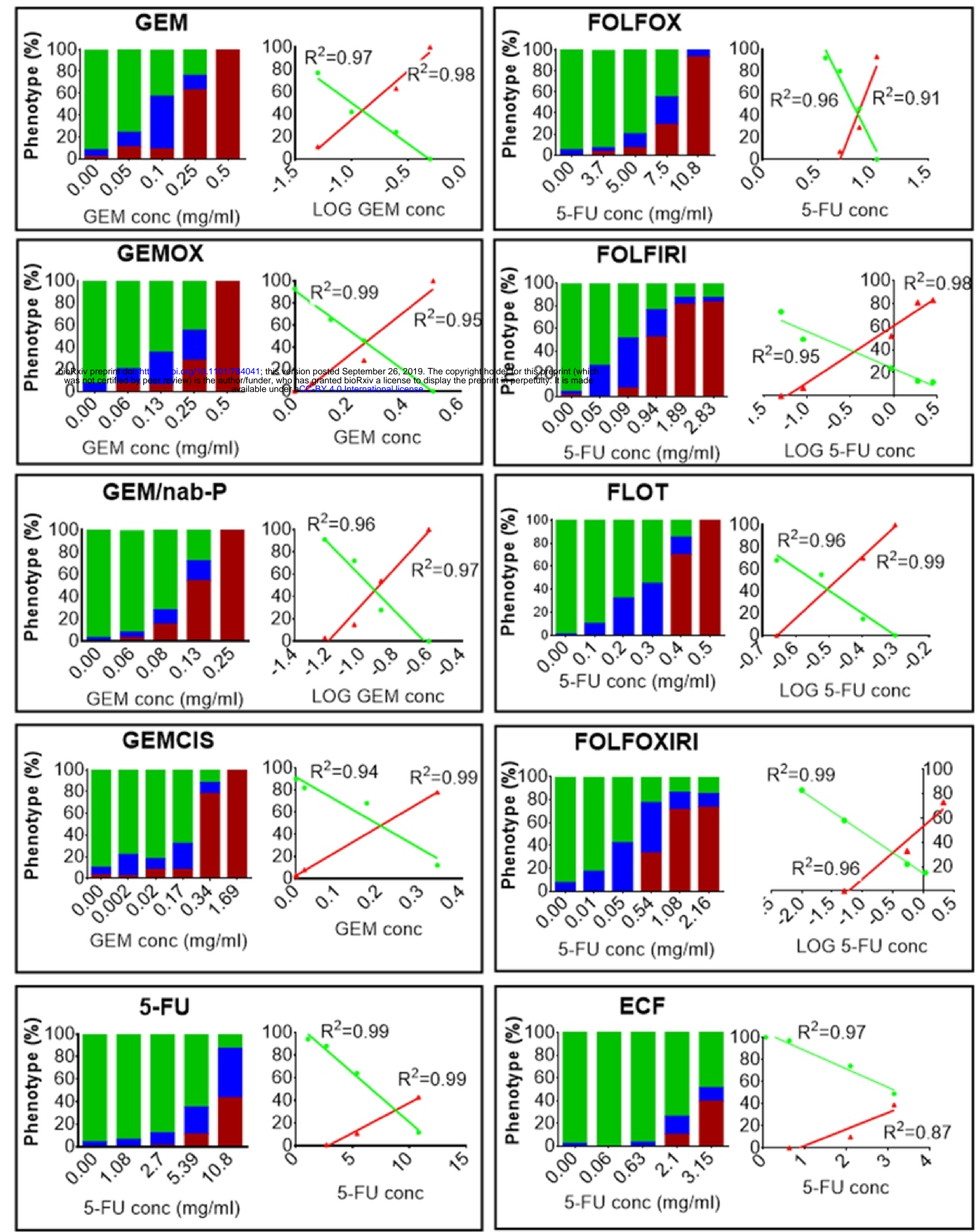

Figure 1 
A

\begin{tabular}{ccc} 
Protocol & EPC / IC50 & EPC / LD25 \\
\hline GEM & 3,35 & 4,41 \\
GEMOX & 1,5 & 2,13 \\
GEM/nab-P & 3,03 & 3,77 \\
GEMCIS & 1,73 & 3,35 \\
5-FU & 0,16 & 0,14 \\
FOLFOX & 0,14 & 0,15 \\
FOLFIRI & 6,21 & 5,03 \\
FLOT & 3,08 & 3,23 \\
FOLFOXIRI & 11,99 & 5,55 \\
FCE & 0,29 & 0,37
\end{tabular}

B

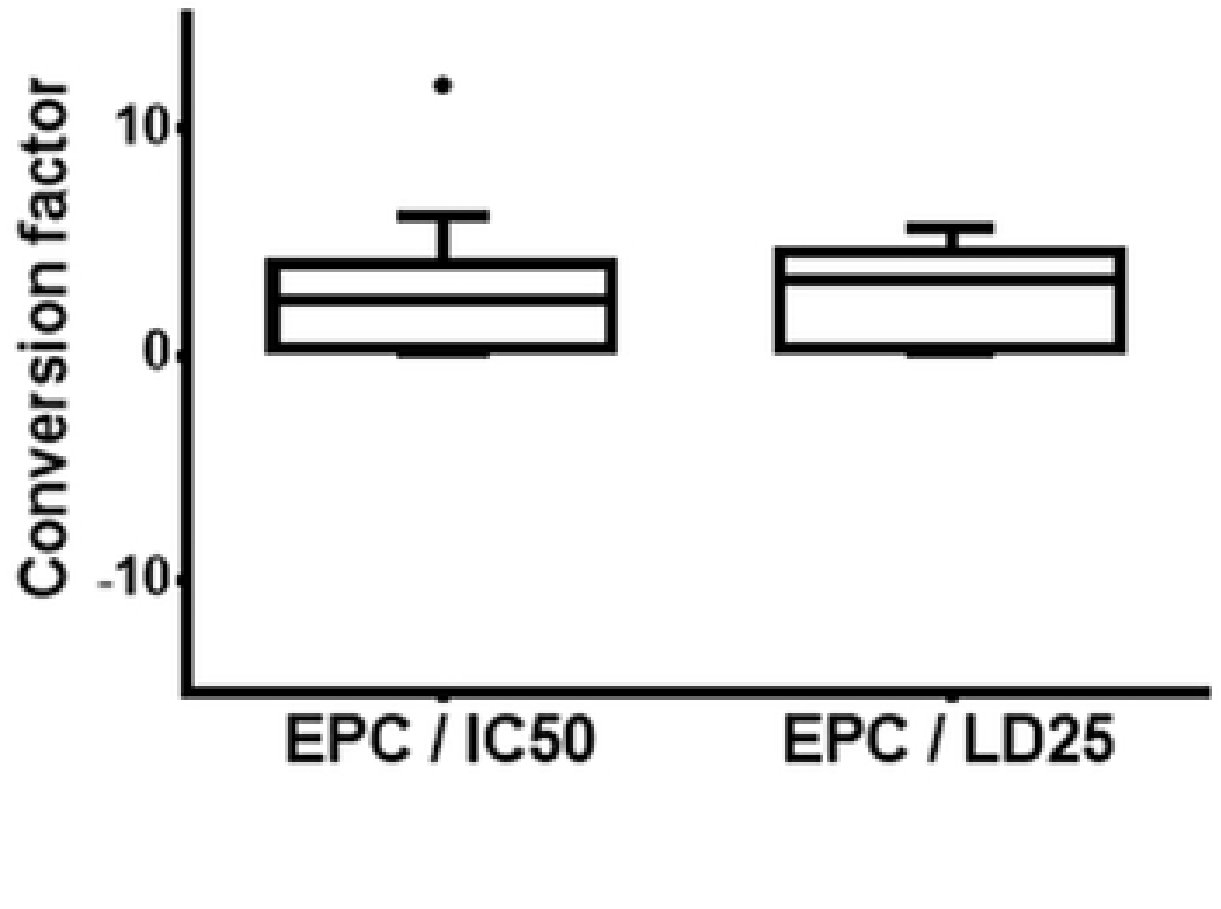

Figure 2 

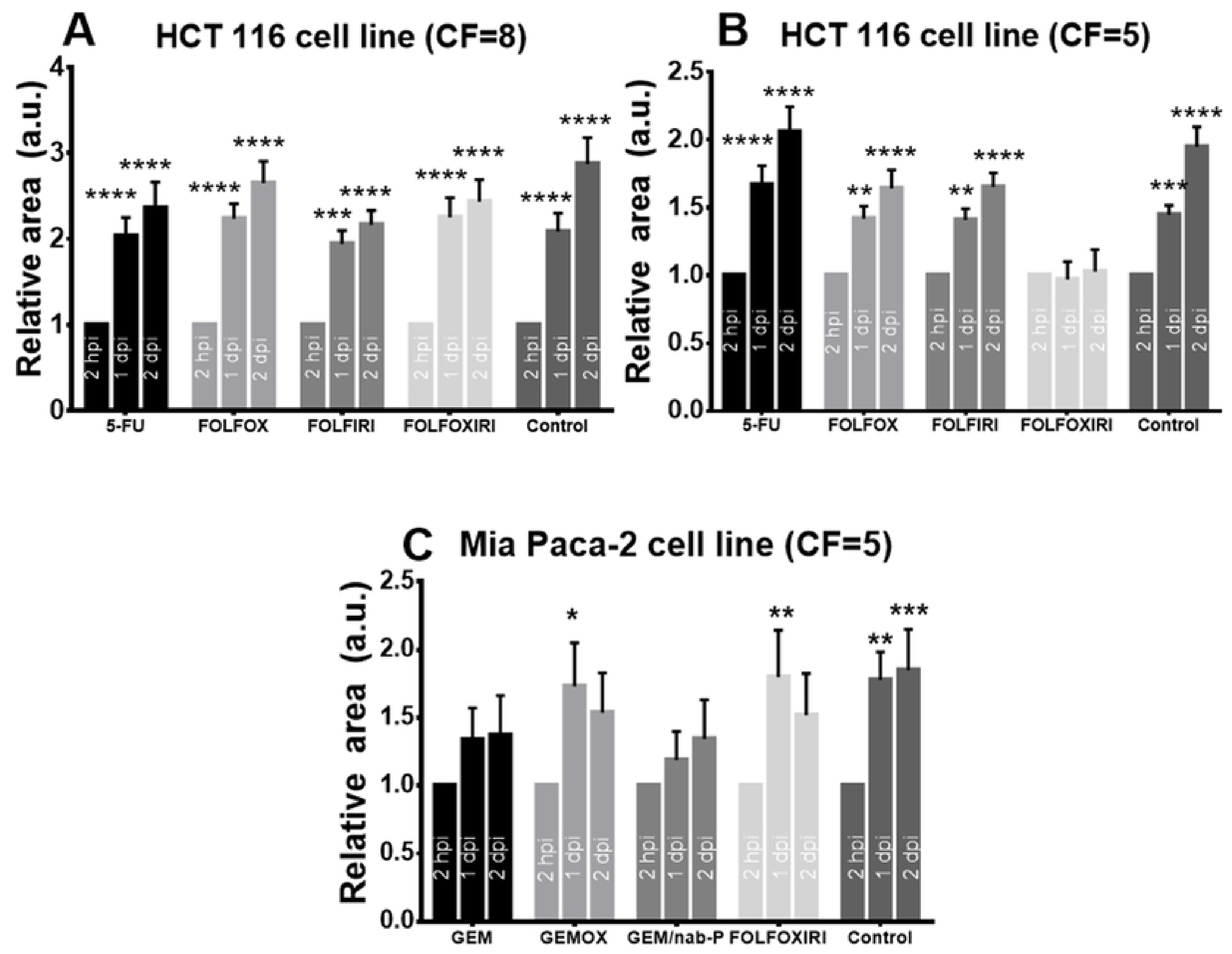

Figure 3 


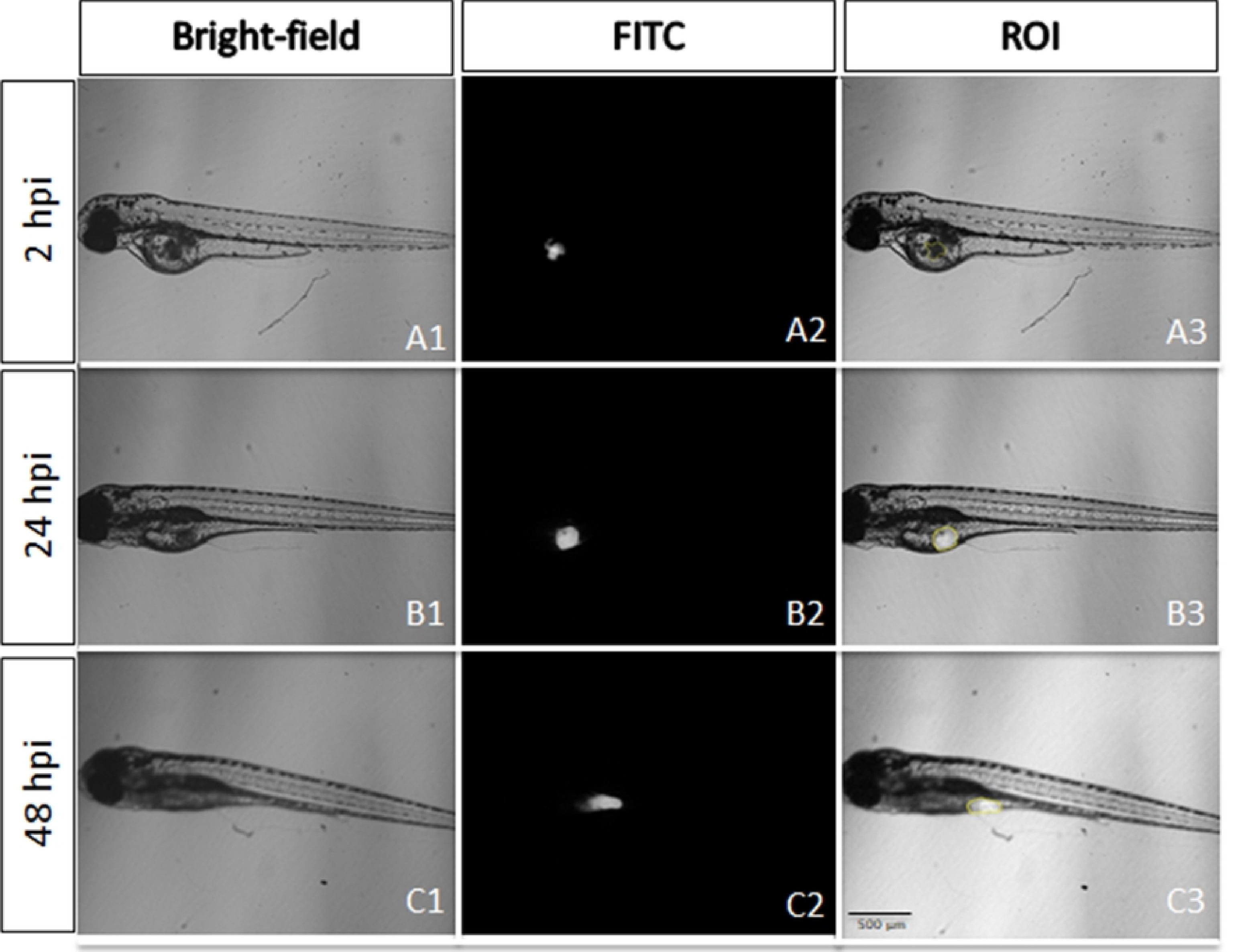

Figure 4 

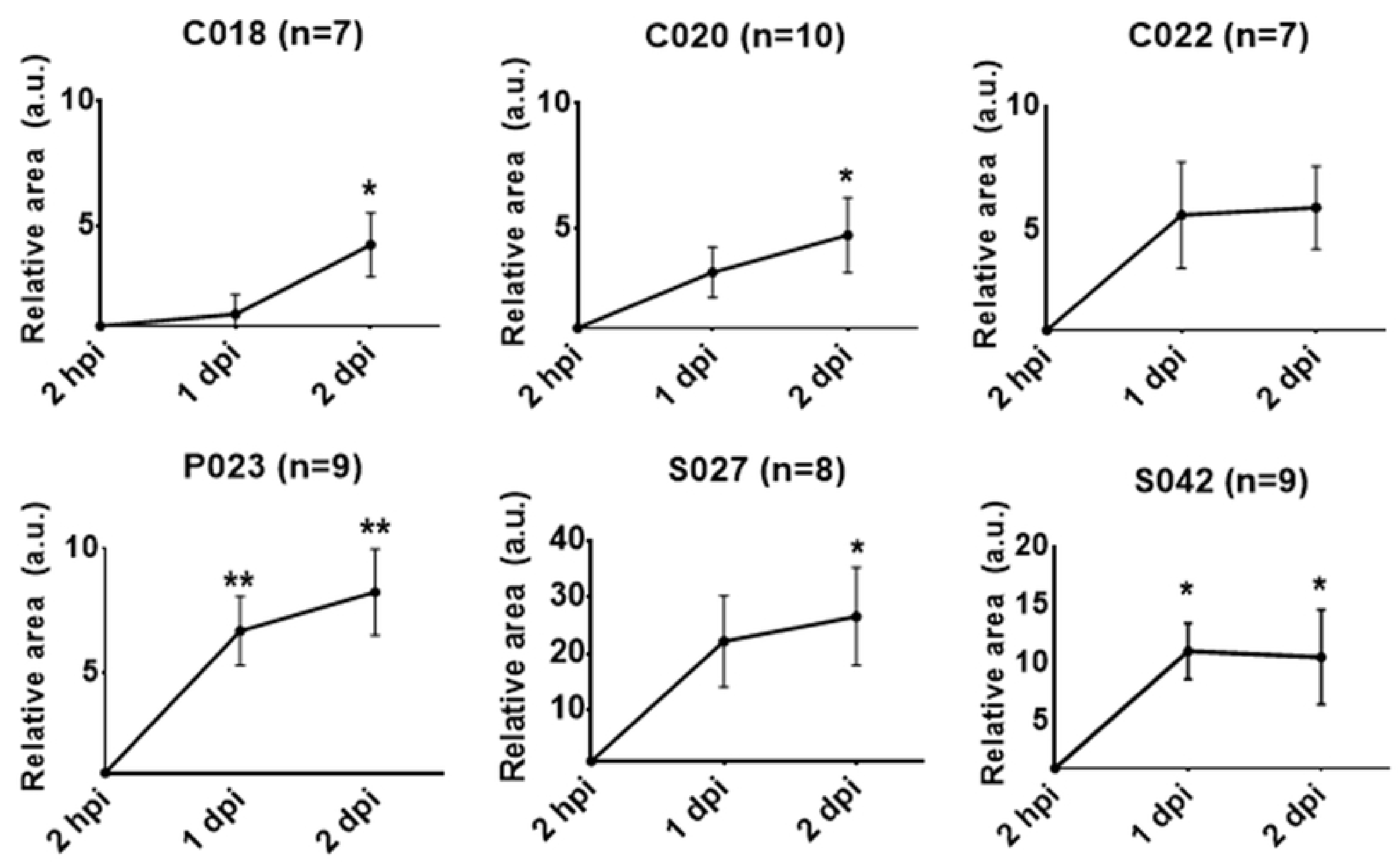

Figure 5 
A

Colon

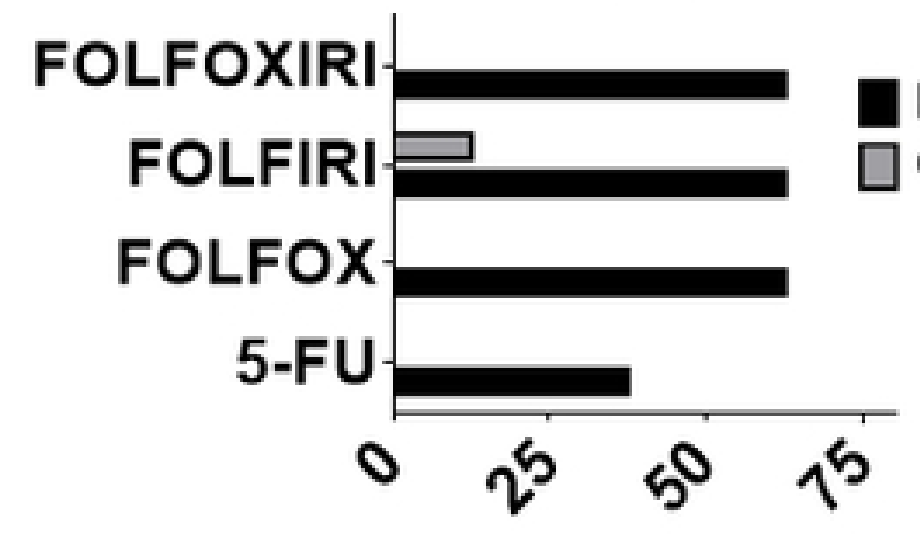

B

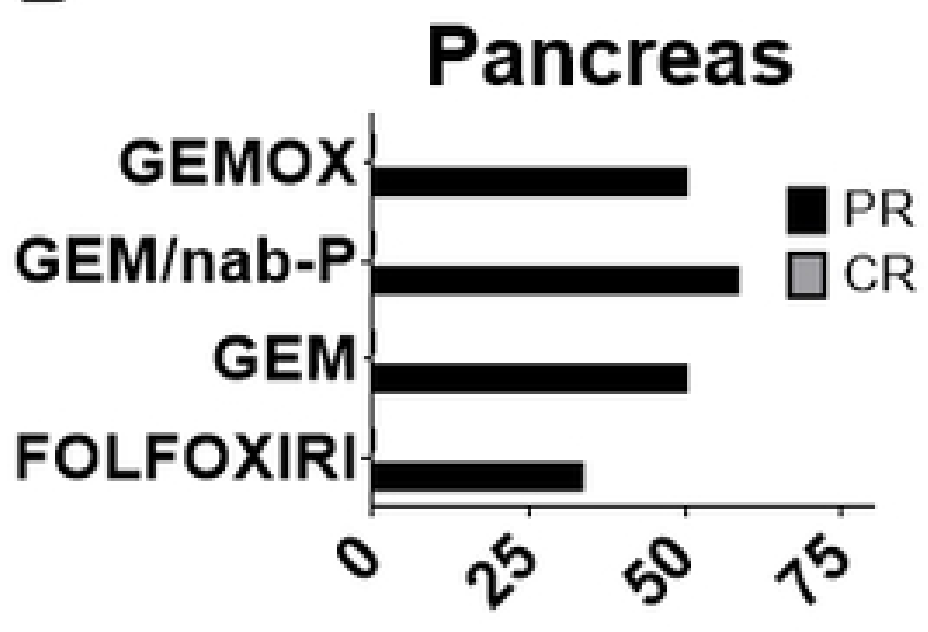

C

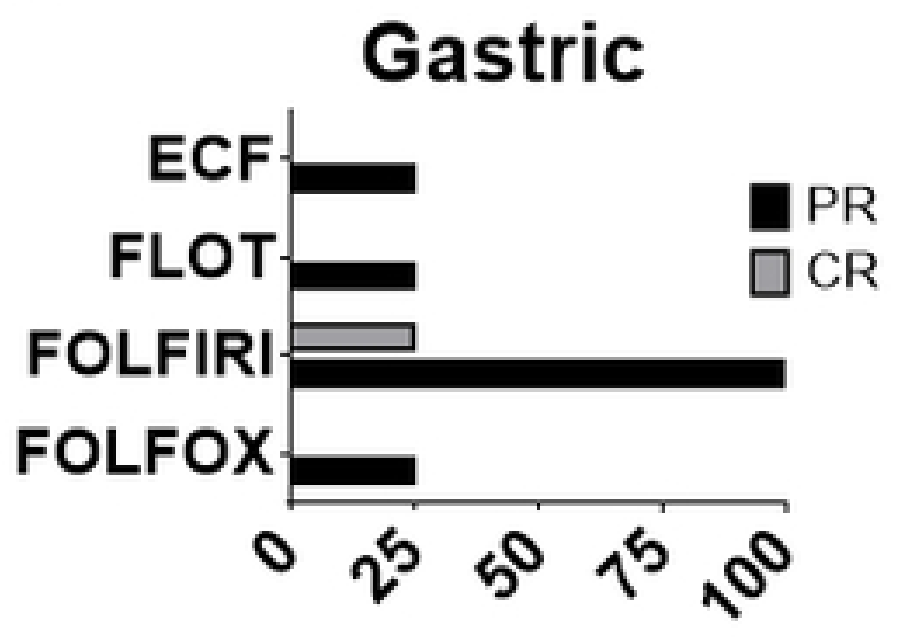

Figure 6 

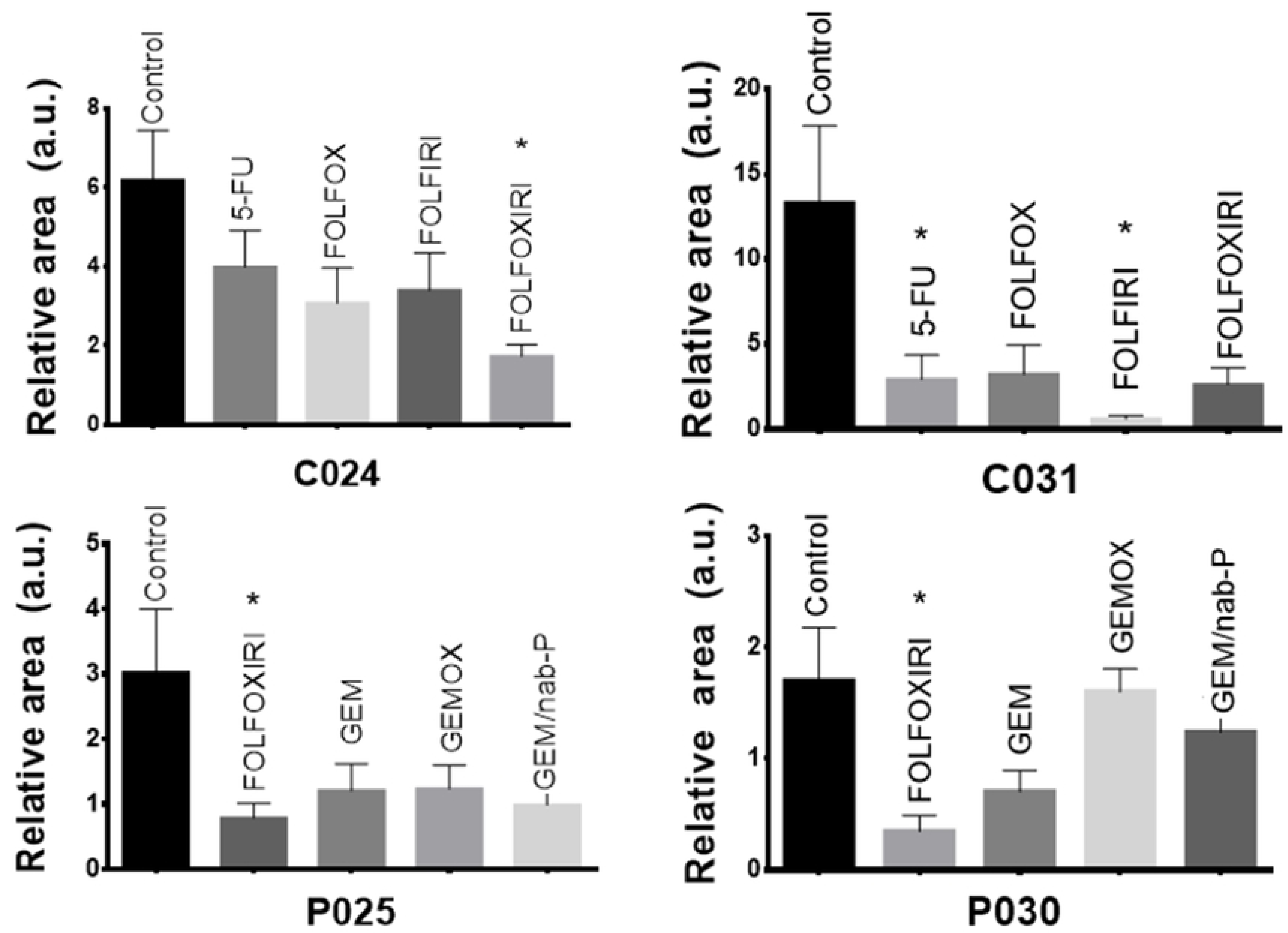

C031

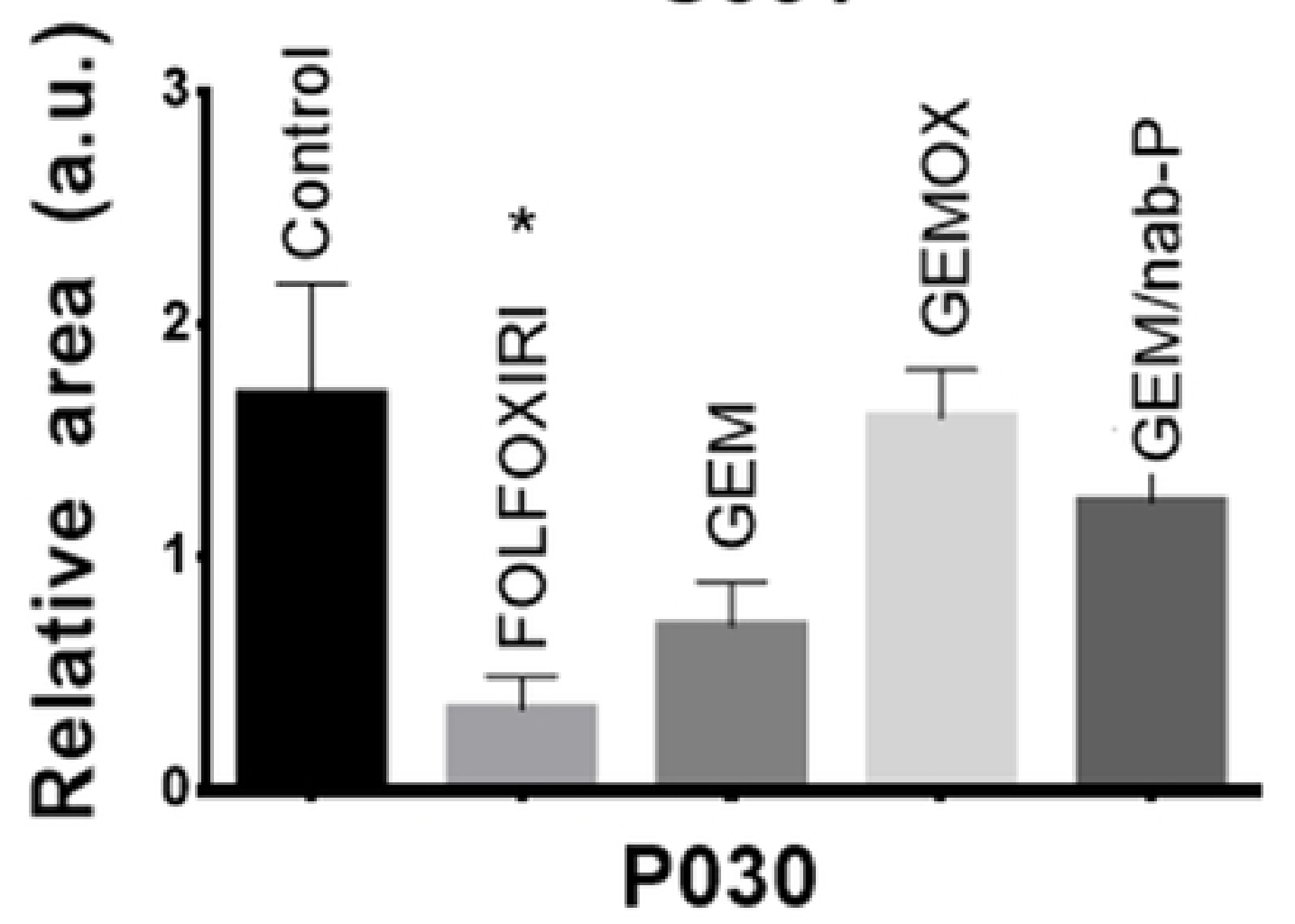

Figure 7 